procerlures have been employed-gastroenterostomy (40 times with 21 recoveries), divulsion (11 times with 7 recoveries), pyloroplasty (8 times with 4 recoveries), and pylorectomy (once with a fatal result). All the deaths can be accounted for by getting the patient too late, and by faulty technique in the operation. These authors consider a gastroenterostomy the most suitable, and, considering the liability of jejunal ulceration to supervene on the anterior variety, advise the use of the posterior form. However, as the duodenum is very mobile in the infant, and as adhesions are never present, some form of gastro-pyloro-duodenostomy might prove a very suitable operative measure. -L. F.

\title{
DISEASES OF THE SKIN.
}

\section{By J. WYLLIE NICOL, M.B., C.M.}

On Lupus Vulgaris Postexanthematicus. By Dr. Franz von Veress (Monatshefte für Praktische Dermatologie, lst .June, 1905). - The writer had recently the opportunity of examining lesions from six different situations in a case of disseminated lupus in a 6 year old boy. The lupus had followed an attack of chicken-pox, which had been preceded three months before by measles.

From his histological findings, and a critical review of the literature of similar cases with regard to the evidence for or against the embolic and inoculation theories, Dr. von Veress draws the following conclusions :-

1. After the acute exanthemata of children-chicken-pox, measles, scarlatina --a disseminated tuberculosis in the form of lupus vulgaris occurs, sometimes during convalescence, but oftener after it is over.

2. All the facts and clinical symptoms, namely, the numerous lesions of the skin, the absence of metastasis of internal organs, the absence of general embolism, the appearance of disseminated lupus after and not during the acute exanthem, the frequent later increase in the number of the lesions, point to an external origin through inoculation.

The only arguments which seem to support the hæmatogenous origin--the multiplicity, and the simultaneous appearance of many foci-could just as well be used in favour of the external origin, because inoculation could take place simultaneously at many sites on the much damaged tender skin of a child.

3. In the histological structure there are absent those features which would support the hæmatogenous origin, namely, participation of the blood-vessels in the formation of the lupus focus, and the occurrence of the nodules in all the layers of the skin.

(In connection with this subject it is of interest to recall a paper on "Multiple Lupus Vulgaris consecutive to Measles," by Dr. H. G. Adamson, in the British Journal of Dermatology, vol. xvi, No. 10. The writer summarised twenty-eight published cases in tabular form, and came to the conclusion that the embolic theory was the most satisfactory one. The most feasible explanation seemed to be that the toxin of measles softened some pre-existing tubercular focus, and gave rise to an embolic shower.)

Xantho-erythrodermia Perstans. By Dr. H. Radcliffe-Crocker (British Journal of Dermatology, April, 1905).- Under the above provisional title Dr. Crocker describes an affection of which he has met ten instances in the last three years, all but one in private practice. His description was drawn up from nine of the cases, all males, which closely resembled each other; the remaining case, a lady, had some important differences.

All of the cases were adults, though some of them were young. The lesions 
were evolved in pale pink or yellowish patches on the limbs and trunk, the face and hands being free or very slightly affected. Generally the patches came out very gradually and in small numbers, and, in the main, symmetrically. As the older patches never went away spontaneously, and fresh ones were continually evoluting at short or long intervals, large areas were evolved, and in the course of years (in one case months) the whole trunk and limbs were crowded with lesions.

For the most part the original patches were discrete, and enlarged but little after their formation, unless they merged into adjoining patches, when handsized or larger areas were found. Individual patches were usually oval or elongated, arranged symmetrically in oblique lines on the back in the direction of the ribs, probably in the lines of fission, more or less horizontal in front, and often, but not always, in vertical lines on the limbs. On the latter, especially the thighs, they not infrequently presented the appearance of streaks formed by the finger, the upper part of the stroke being abrupt, and the lower shading off. This could also be seen on the trunk. The majority of the single patches ranged from 1 inch to 3 inches in their longest diameter; the borders were not very well defined, nor raised above the rest, but there was no difficulty in discerning the morbid from the healthy skin. They were not raised above the surface, but were rather deep in the cutis. Infiltration could often be distinctly felt, but in recent and small patches it was imperceptible, and occasionally they looked like mere stains. Their colour was pale pink or yellowish. The surface was smooth on the trunk, but was often slightly rough on the arms and thighs, and below the knees sometimes distinctly rough or scaly. The patches were never so marked on the upper as on the lower limbs, the palms were always free, and the backs of the hands were generally unaffected. The face was nearly always free, though in one case there were a few faint patches. There was little to suggest that the disease was inflammatory, and itching was quite absent in most of the cases.

The initial site for the lesion varied; the thighs were most frequently first affected, the legs next, and then the trunk. The lower limbs were generally more affected than other parts.

The duration varied from a few months to over ten years. The majority of the patients had otherwise excellent health. There appeared to be no tendency to spontaneous involution, but treatment with 15 grain doses of salicin and the application of 10 per cent vasogen iodine seemed to cure two cases and improve several others.

The female case, aged 47 years, resembled the others in gradual evolution, long duration, absence of itching, in the persistence of old patches with continued evolution of new ones, in its limitation to the covered parts, and in the general good health of the patient. The differences were in the patches being distinctly scaly. Though the scales were small and powdery in most parts, they were, as usual, rather larger and more abundant on the legs. The patches were more decidedly red than in the other cases. The disease had been present for ten years, and various forms of treatment had been employed without benefit. Dr. Crocker tried Röntgen rays, and the parts exposed cleared up; so in future he intends to use the rays.

No cause could be made out, and Mr. Pernet did not find any explanation on examining a portion of skin from one of the cases histologically, except that the appearances of the blood-vessels suggested a general blood condition. The stratum granulosum was atrophied or absent, the stratum lucidum absent, except for occasional traces. The epidermis showed slight œedema. In the corium the vessels were dilated, with cellular infiltration about them. There was slight cedema, and the elastin seemed reduced in quantity, but stained normally.

Dr. Crocker compares the affection with a seborrhoïde, urticaria pigmentosa, parapsoriasis, and Brocq's "erythrodermie pityriasique en plaques disseminées," and concludes that at present he must regard it as a separate dermatosis. 